\title{
Electromechanical Coupling Approach for Traction Control System of Distributed Drive Electric Vehicles
}

\author{
Xiang $\mathrm{Gao}^{1}$, Cheng $\mathrm{Lin}^{1,2}$ \\ ${ }^{1}$ National Engineering Laboratory for Electric Vehicles, Beijing Institute of Technology, Beijing 100081, China \\ ${ }^{2}$ Collaborative Innovation Center of Electric Vehicles in Beijing, Beijing Institute of Technology, Beijing 100081, China
}

\begin{abstract}
This paper proposed an electromechanical coupling approach based on sliding mode control (SMC) for traction control systems (TCS) of distributed drive electric vehicles (DDEVs). Since all wheel torque can be controlled continuously and independently, the TCS could be precisely applied on DDEVs. However, normal TCS would cause the waste of motor torque and road adhesion on the special working conditions. To solve this problem, the SMC was utilized based on the optimal slip rate calculated by road adhesion condition recognition and the electromechanical coupling (EC) approach was proposed to deliver part of torque from the motor of the higher speed. Simulation results based on dSPACE simulator showed that the proposed strategy can improved the dynamic performance and passability of the DDEVs.
\end{abstract}

\section{Introduction}

With the increasing environmental pollution and road safety, the DDEVs have ignited wide-spread interest because of the ability to reduce pollution and make driving safer [1]. Vehicles would be unsafe under some special road condition, such as slippery road and climbing condition. TCS mainly involve two aspects of research: road adhesion condition recognition and torque control strategies. The former can be divided into two categories: cause-based and effect-based [2]. The effectbased method is an indirect estimation method, which estimated the current road adhesion condition by measuring the parameters of vehicle motion state changes caused by the change of road adhesion coefficient. This method is low-cost and easy to realize due to the characteristic of DDEVs and has been widely used in society.

Since the DDEVs have the great advantages in control, the TCS has been making continuous progress. The common strategies contained PID, fuzzy control and SMC [3-5]. However, they were acceptable for simple conditions and limited by single motor torque output. This paper combined the SMC and EC to make the full use of the total motor torque for better dynamic performance.

\section{Vehicle model}

\subsection{Description of new structure of DDEV}

A new structure of DDEV were shown in Fig.1. It contains motors, reducers and half-shaft. Compared with the in-wheel motor drive, it can reduce the poor drive comfort caused by the increase of the unsprung mass of the vehicle, and has good heat dissipation performance. In addition, two power coupling devices were integrated in the front and rear reduction gearboxes to realize the coupling of the power between the wheels and axles to improve the dynamic performance and passability.

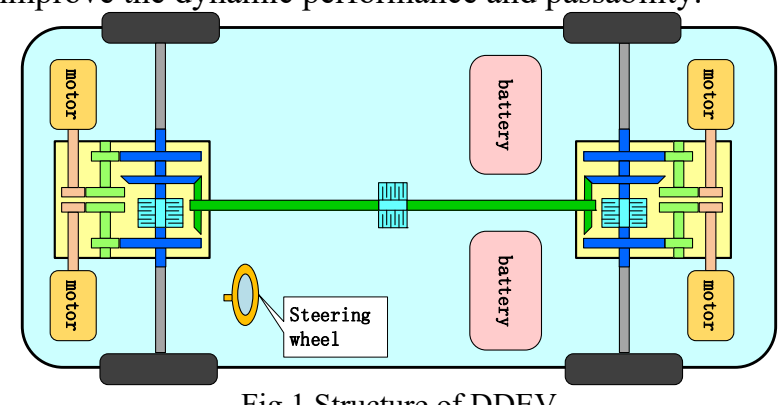

Fig.1 Structure of DDEV

\subsection{Vehicle dynamic model}

This paper mainly focused on vehicle longitudinal dynamics. In order to facilitate the problem analysis, the dynamic model was appropriately simplified. Assuming that there was no steering operation, and the difference of ground forces on the left and right wheels can be ignored when driving straight, the simplified $1 / 4$ vehicle model is obtained as Fig.2. 


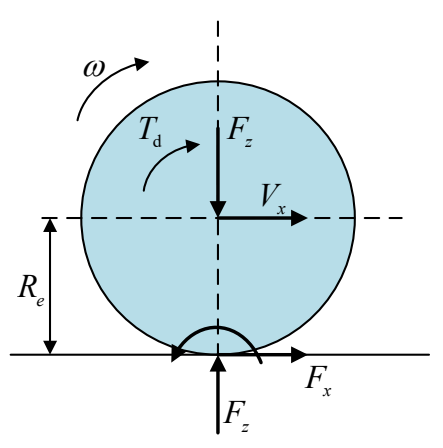

Fig. 2 1/4 vehicle dynamic model

The dynamic model can be described as follow:

$$
\begin{aligned}
& m \dot{V}_{x}=F_{x}-f F_{z} \\
& J_{\omega} \dot{\omega}=T_{d}-F_{x} R_{\mathrm{e}}-f F_{z} R_{\mathrm{e}} \\
& \lambda=\frac{\omega R_{e}-V_{x}}{\omega R_{e}}
\end{aligned}
$$

Where $m$ is the vehicle mass; $V_{x}$ is the longitude velocity; $F_{x}$ is the tangential force between tire and ground; $f$ is the rolling resistance coefficient; $F_{z}$ is the vertical load; $J_{\omega}$ is the wheel moment of inertia; $\omega$ is the wheel speed; $T_{d}$ is the wheel torque output; $R_{\mathrm{e}}$ is the rolling radius, $\lambda$ is the slip rate.

Due to the existence of acceleration resistance and slope resistance, the vertical load can be simply described as follow:

$$
\left\{\begin{array}{l}
F_{z 1}=F_{z 2}=\frac{m g b \cos \alpha}{2 L}-\frac{m g h_{g} \sin \alpha}{2 L}-\frac{m \dot{V}_{x} h_{g}}{2 L} \\
F_{z 3}=F_{z 4}=\frac{m g a \cos \alpha}{2 L}+\frac{m g h_{g} \sin \alpha}{2 L}+\frac{m \dot{V}_{x} h_{g}}{2 L}
\end{array}\right.
$$

Where $a$ and $b$ is the distance from front or rear axle to the centroid of the vehicle (CG); $L$ is the wheelbase; $h_{g}$ is the height of the centroid of the vehicle; $\alpha$ is the road slope.

\section{TCS Strategy}

\subsection{Road adhesion condition recognition}

The relationship between the road adhesion coefficient and the tire slip rate has a certain function based on recent research. And the specific function curves of different pavements are different, but the shapes are similar. Therefore, the identification of road adhesion conditions is also the identification of road curve shape. This function is based on the six different type of Burckhardt standard road [6]:

$$
\mu(\lambda)=c_{1}\left(1-e^{-c_{2} \lambda}\right)-c_{3} \lambda
$$

Where $c_{1}, c_{2}, c_{3}$ are the fitting characteristic parameters of different standard road. The optimal slip rate and maximum adhesion coefficient of different standard road can be calculated according to three parameters.

According to the wheel speed, angular acceleration, motor torque and vertical load, the real-time slip rate and adhesion coefficient can be calculated. The obtained results were taken as the input of fuzzy control and transformed into corresponding membership function. Finally, the fuzzy controller outputs the similarity degree between the current road slip rate and the corresponding $(\mu, \lambda)$ of the six type of road database.

$$
\lambda_{\text {opt }}=\frac{w_{1} \lambda_{\text {opt } 1}+w_{2} \lambda_{\text {opt } 2}+w_{3} \lambda_{\text {opt } 3}+w_{4} \lambda_{o p t 4}+w_{5} \lambda_{o p t 5}+w_{6} \lambda_{o p t 6}}{w_{1}+w_{2}+w_{3}+w_{4}+w_{5}+w_{6}}
$$

\subsection{SMC strategy}

The main research of this paper is the TCS, so the wheel drive torque can be solved by sliding mode variable structure control based on the optimal slip rate. The slip rate was controlled near the optimal slip rate, so that the vehicle can obtain the maximum longitude force on the current road. The sliding surface was designed as follow:

$$
s=\lambda-\lambda_{\text {opt }}
$$

Using an improved exponential approaching law [7], the approaching speed is proportional to $s^{2}$, which has better dynamic response and stability than the original exponential approaching law.

$$
\dot{s}=-\eta s^{2} \operatorname{sgn}(s)-k s
$$

As $\eta$ is small and $k$ is large, the system has a larger approach speed when far from the sliding surface, but has a smaller speed when moves to the vicinity of the sliding surface. Therefore, the controller possesses a fast convergence speed and a small chattering. In addition, the controller uses a sign function, which is a discontinuous function that affects the chattering of the system. In order to weaken the chattering effect caused by the sign function, the following function is used instead, as shown below:

$$
f(s)=\frac{1-e^{-q s}}{1+e^{-q s}}, q>0
$$

Combined formulas (1-4) and (7-8), the final output torque control law of sliding mode controller is

$T_{d i}=\frac{J_{\omega} \omega^{2} \mathrm{R}_{e}}{V_{x}}\left(\frac{\mu(\lambda) F_{z} \mathrm{R}_{e}+f F_{z} \mathrm{R}_{e}}{J_{\omega} \omega^{2} \mathrm{R}_{e}}+\frac{\mu(\lambda) F_{z} \mathrm{R}_{e}-f F_{z} \mathrm{R}_{e}}{m \omega \mathrm{R}_{e}}-\eta s^{2} \frac{1-e^{-q s}}{1+e^{-q s}}-k s\right)$

In addition, the constraints of motor torque and corresponding electromechanical coupling torque need to be taken into account.

\section{Results and Discussions}

In this section, the performance of the proposed strategy is tested based on dSPACE simulator. The parameters of the DDEV are shown in Table 1

Table 1 DDEV parameters

\begin{tabular}{|l|l|l|l|}
\hline Parameters & Values & Parameters & Values \\
\hline Vehicle Mass & $1523 \mathrm{~kg}$ & $\begin{array}{l}\text { Distance from front } \\
\text { axle to CG }\end{array}$ & $1.28 \mathrm{~m}$ \\
\hline Wheelbase & $1.53 \mathrm{~m}$ & $\begin{array}{l}\text { Dimensionless } \\
\text { coefficient }\end{array}$ & 0.3 \\
\hline
\end{tabular}




\begin{tabular}{|l|l|l|l|}
\hline $\begin{array}{l}\text { Vehicle } \\
\text { Moment of } \\
\text { Inertia }\end{array}$ & $\begin{array}{l}1558 \\
\mathrm{Nm}\end{array}$ & Front area & $1.95 \mathrm{~m}^{2}$ \\
\hline Wheel radius & $0.354 \mathrm{~m}$ & Transmission ratio & 8 \\
\hline $\begin{array}{l}\text { Distance from } \\
\text { front axle to } \\
\text { CG }\end{array}$ & $1.19 \mathrm{~m}$ & Friction coefficient & 0.01 \\
\hline
\end{tabular}

\subsection{Slippery road test}

On this condition, the steering angle was set to zero and the road adhesion coefficient was set to 0.2, DDEV started at an initial velocity of $0.5 \mathrm{~m} / \mathrm{s}$ for prevention of sudden change of starting slip rate. It can be seen from

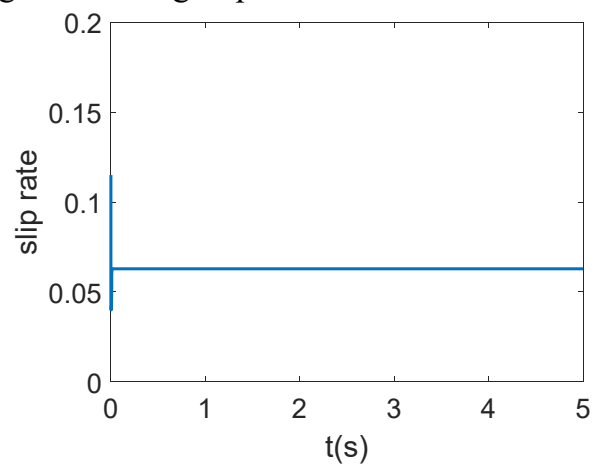

(a) Identification results of optimal slip rate Fig.3 Results of different strategy

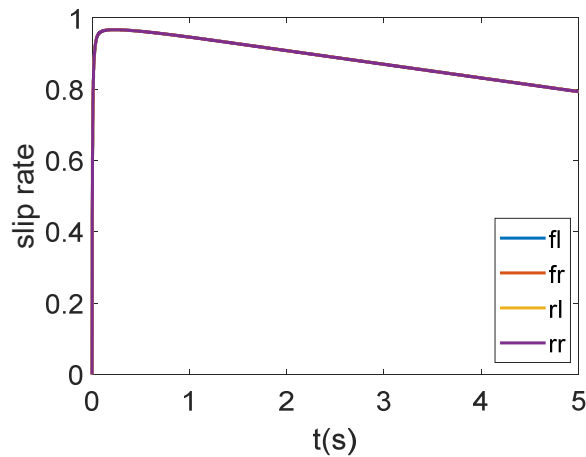

(a) Slip rate without control

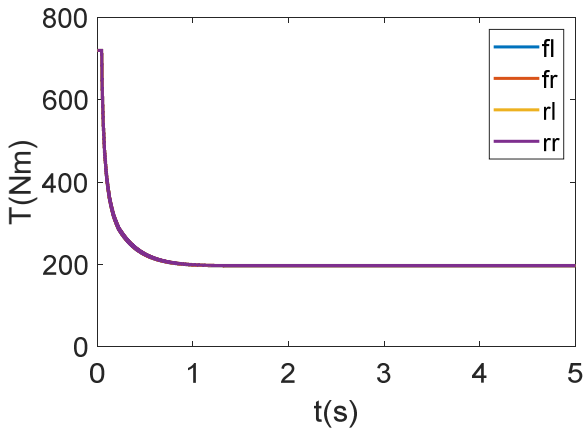

(c) Wheel torque without control

Fig. 4 Comparison of different strategy

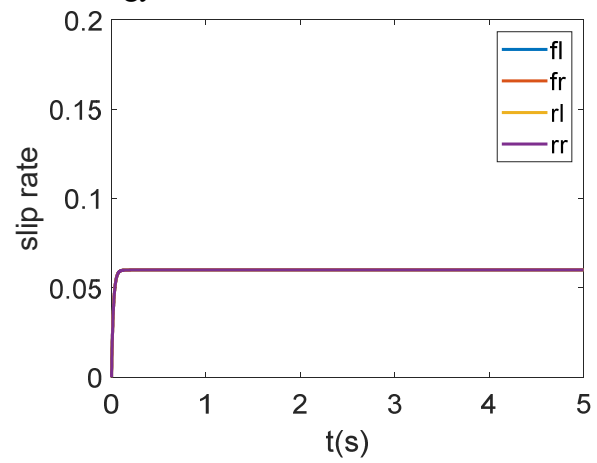

(b) Slip rate under SMC

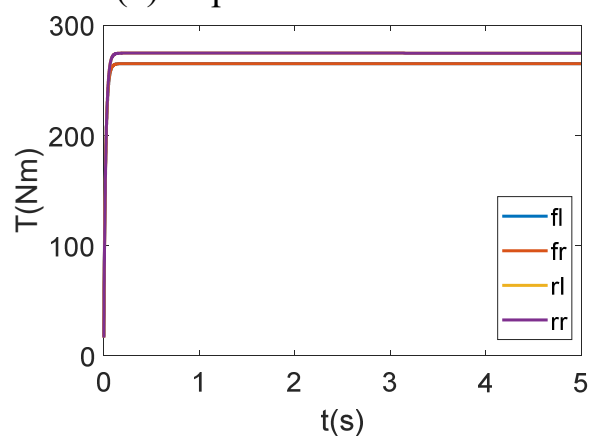

(d) Wheel torque under SMC
Fig.3(a) that the estimated value of optimal slip rate obtained by fuzzy controller identification was 0.063 , and the error was really small compared with the real value of 0.06 . The result from Fig.3(b) presented that the speed in $5 \mathrm{~s}$ without control was $6.6 \mathrm{~m} / \mathrm{s}$, while the speed of SMC was $9.2 \mathrm{~m} / \mathrm{s}$, which was increased by $40 \%$. Fig. 4 the comparison of slip rate and corresponding torque. It can be seen that SMC can follow the optimal slip rate 0.06 quickly and stably, and adjust the output torque to improve the dynamic performance of the vehicle.

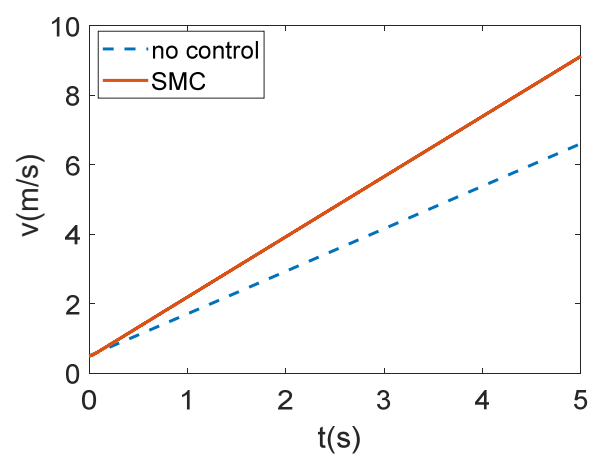

(b) Velocity comparison

value of 0.12 . The result from Fig.5(b) presented that the speed in $5 \mathrm{~s}$ without control was $10.8 \mathrm{~m} / \mathrm{s}$, while the speed of SMC was $11.7 \mathrm{~m} / \mathrm{s}$, which was increased by $8.3 \%$. However the speed of the SMC and EC was $12.1 \mathrm{~m} / \mathrm{s}$, which was increased by $12.0 \%$ compared with no control and $3.4 \%$ compared with SMC. Fig. 6 showed the comparison of slip rate and corresponding torque. 


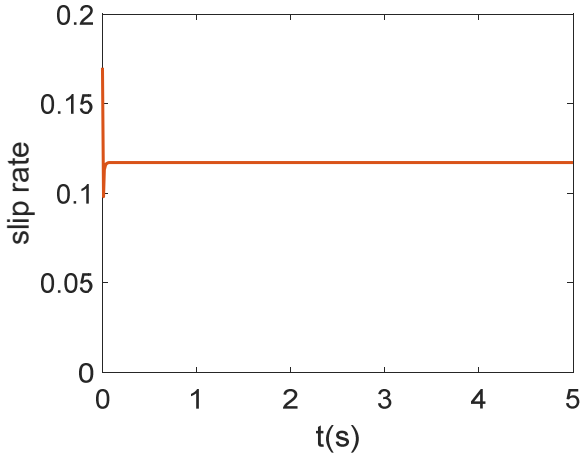

(a) Identification results of optimal slip rate

Fig.5 Results of different strategy

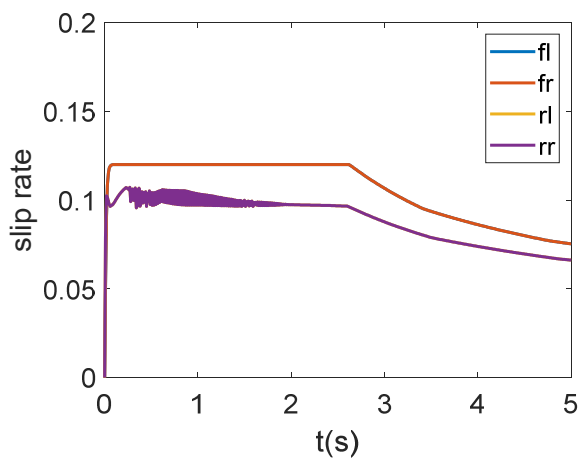

(a) Slip rate under SMC

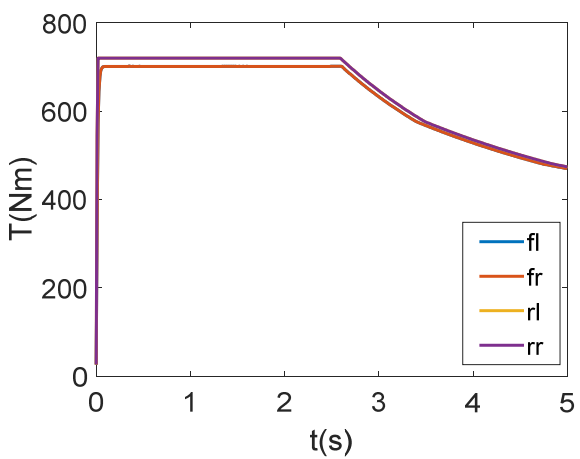

(c) Wheel torque under SMC

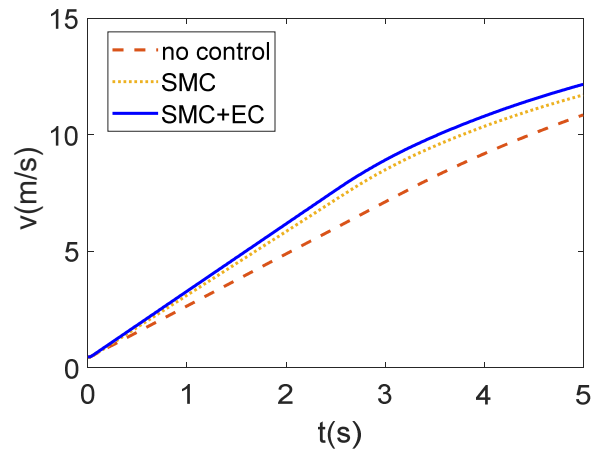

(b) Velocity comparison

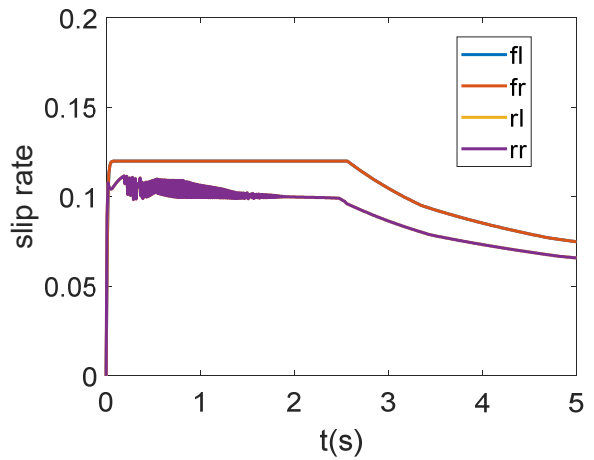

(b) Slip rate under $\mathrm{SMC}+\mathrm{EC}$

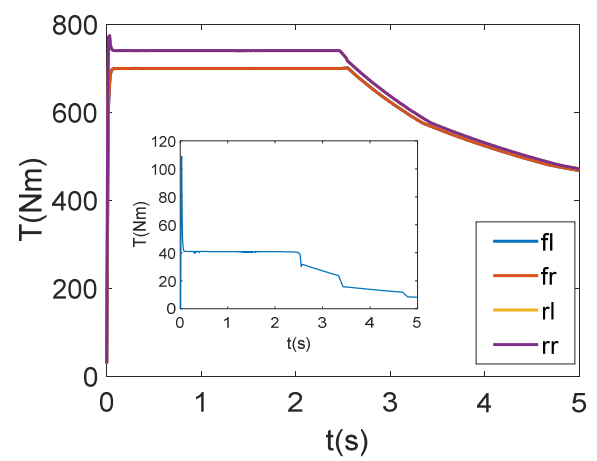

(d) Wheel torque under SMC+EC

Fig.6 Comparison of different strategy

From the Fig.6(c) and (d), it can be seen that due to the increase of the rear axle load, the wheels of the rear axle failed to make full use of the road adhesion capacity when the electromechanical coupling control was not applied, and the driving torque was insufficient. While the maximum torque of the front axle was not reached and the remaining driving torque can be transferred to the rear axle wheels, so as to improve the dynamic performance. Because of the configuration constraints, inter-axle coupling needs to achieve torque transmission between the wheels on both sides of one axle through inter-axle coupling. Therefore, the inter-wheel coupler transmits half of the torque of the inter-axle coupler. The torque transmission curve of the coupler was shown in Fig.6(d), which verified the effectiveness of the electromechanical coupling control.

\section{Conclusion}

Based on the results and discussions presented above, the conclusions are obtained as below:
(1) A new configuration of DDEV was proposed to realize the electromechanical coupling for making full use of the total driving force.

(2) The electromagnetic coupler was applied to transfer the torque from the redundant driving force side to the insufficient driving force side. The slip rate of each wheel can be controlled near the optimal slip rate with convergence and strong anti-interference ability under different working conditions. It can make full use of the road adhesion ability and the coupling ability between motors to improve the dynamic performance and passability of the vehicle.

\section{Acknowledgments}

This work was supported by the National Natural Fund (51575044) and National Key R\&D Program of China (2017YFB0103801). 


\section{References}

1. Yu Zhuoping, Feng Yuan, Xiong Lu. Review on Vehicle Dynamics Control of Distributed Drive Electric Vehicle[J]. Chinese Journal of Mechanical Engineering, 2013, 49: 105-114 (in Chinese).

2. Ahn Changsun, Peng Huei, Tseng H. Eric. Robust estimation of road friction coefficient using lateral and longitudinal vehicle dynamics[J]. Vehicle System Dynamics, 2012, 50: 961-985.

3. Khosravani Saeid, Jalali Milad, Khajepour Amiret al. Application of Lexicographic Optimization Method to Integrated Vehicle Control Systems[J]. IEEE transactions on Industrial Electronics, 2018, 65(12): 9677-9686.

4. Ding Xiying, Li Lin, Yu Hua, et al. Integrated DYC/ASR-based variable universe fuzzy control for electric vehicles[J]. Automotive Engineering, 2014, 36(5): 527-531 (in Chinese).

5. Song Pan, Zong Chang-fu, Tomizuka Masayoshi. A terminal sliding mode based torque distribution control for an individual-wheel-drive vehicle[J]. Journal of Zhejiang University, 2014, 15(9): 681693 (in Chinese).

6. Canudas-de-Wit Carlos, Tsiotras Panagiotis, Velenis Efstathioset al. Dynamic friction models for road/tire longitudinal interaction[J]. Vehicle System Dynamics, 2003, 39(3): 189-226.

7. Li Xuedong. Study on traction control algorithm for four-wheel drive electirc vehicle under low adhesion conditions[D]. Hefei University of Technology, 2019 (in Chinese). 